\title{
Thermodynamic Geometrical Analysis on Properties of Black Holes
}

\author{
Xiang Xu \\ China West Normal University, Nanchong, Sichuan, 637009, China
}

\author{
Keywords: Thermodynamic geometry, Black holes, Properties
}

\begin{abstract}
Thermodynamic geometry is a special method for the research of black holes. Following that achievements in Hawking radiation of black holes and black hole entropy are obtained and the relationship between black holes and thermodynamics is figured out, more and more people are obsessed with thermodynamic research of black holes. This paper mainly introduces the basic theory of black-hole thermodynamic geometry, and discusses the thermodynamic geometrical properties of RN.
\end{abstract}

\section{Introduction}

Black-hold thermodynamics, also called black-hole mechanics, refers to a new theory produced in the process of adopting the General Relativity Theory, in combination with the fundamental laws of the original thermodynamics, to research black holes. Though people are unable to clearly interpret the theory, it is greatly helpful for people to figure out the correlation among the thermodynamics, the General Relativity Theory and the quantum theory. Superficially, black-hole thermodynamics just relates to the restrictions of the fundamental laws of the thermodynamics on behaviors of black holes. Yet its significance is far more than this. This paper mainly analyzes the properties of black holes from a thermodynamic geometrical angle.

\section{Key Elements of Black-hole Thermodynamics}

\section{No-hair Theorem}

The "hair" is a metaphor for information. The only three externally observable classical parameters in black holes are mass, electric charge, and angular momentum. To put it other way, all information other than mass, electric charge, and angular momentum about the matter which is falling into a black hole disappears. Information is equivalent to negentropy, and the process that a matter is falling into a black hole can be regarded as an increasing process of entropy accordingly. According to the definition of black hole, all matters are likely to fall into it and unable to escape, without an exception. It is true for even radiation. Any radiation falling into a black hole will be absorbed and becomes unable to reflect. In physics, the object that absorbs radiation and disable it to reflect is called black body. As a simple object, the properties of black hole depends solely on temperature. In other words, the spectrum of radiation from a black hole depends on temperature, namely thermal radiation.

\section{Area Theorem}

Hawking has proven the area theorem based on the "cosmic censorship hypothesis" and strong energy condition, namely: the horizon area of black hole is a non-decreasing function of time $(\delta A>0)$. In this theorem, the strong energy condition is $\rho+3 P>0$. Wherein, $\mathrm{p}$ refers to pressure produced by stress, and $\rho$ to matter density. This condition shows that stress will never negatively derivate from zero too much. Most matters can meet this condition. 
The area theorem reminds people of the concept of entropy in the thermodynamics. In physics, apart from entropy and its derived quantities, there is no time unidirectional physical quantity. In thermodynamics, entropy $S$ will not decrease in adiabatic process, namely $\delta S \geq 0$. This is the second law of thermodynamics. According to the area theorem, if two black holes rationally merge, the area increases; yet black hole cannot be disintegrated, of which both the process and result don't conform to the area theorem.

\section{Four Laws of Black Hole Thermodynamics}

The four laws of black hole thermodynamics are: (1) The zeroth law: The horizon has constant surface gravity for a stationary black hole, $\kappa_{+}$; (2) The first law: $d M=\frac{\kappa_{+}}{8 \pi} d A_{+}+\Omega_{+} d J+V_{+} \mathrm{d} Q$; (3) The third law: The horizon area is non-decreasing function of time, $d A_{+} \geq 0$; (4) It is not possible to form a black hole with vanishing surface gravity.

\section{Thermodynamic Geometry Introduction}

As early as 1975, the standard geometrical thought was introduced into thermodynamics, and the second derivative of related functions was put forward. This, however, is of little physical significance. Thereafter, a new metric was introduced, namely:

$$
g_{\mathrm{ab}}^{R}=\frac{\partial^{2}}{\partial X^{a} \partial X^{b}} S(x),(a, b=1,2,3, \ldots n)
$$

It is of considerable significance to the thermal balance wave theory. Besides, it was linked with the former metric via related laws, which is called conformal transformation. Since the metric was put forward, lots of research had been carried out, and it is found to have information of phase structure of thermodynamic system through model research. At the critical point or in phase change process, the curvature scalar of the system appears diverging.

\section{Thermodynamic Geometrical Discussion on Properties of RN Black Hole}

For now, most of the information on black hole is unknown. So are the thermodynamic model or statistical mechanical model relating thereto. Only a few of black holes become known through the superstring or $\mathrm{M}$ theories. Ferrara et al. had learned about it from a thermodynamic geometrical angle in the thermodynamic research of black hole for the first time. This method was subsequently used in black hole researches in different dimensions.

\section{Weinhold's Geometry}

Weinhold's Geometry refers to Hessian matrix for which the metric is defined as internal energy for energy representation, expressed as:

$$
g_{i, j}^{w}=\partial_{i} \partial_{j} M\left(U, N^{a}\right) \text {; }
$$

Wherein, $\mathrm{N}^{\mathrm{a}}$ refers to the selected thermodynamic variable. After the Weinhold's Geometry was put forward, successive researches on its various physical properties like thermodynamic length and space-time property were performed, such as Riemannian geometrical construction. Through researches, the Weinhold's Geometry was found to have some defects, namely it is impossible to give a physical interpretation of metric structure based on the Weinhold's Geometry.

\section{Ruppeiner's Geometry}

In the Ruppeiner's Geometry, the Riemannian geometry is introduced to equilibrium space, and metric is defined to be the second partial derivative of entropy:

$$
g_{i, j}^{R}=-\partial_{i} \partial_{j} S\left(U, N^{a}\right)
$$


According to the Ruppeiner geometric method, the curvature scalar obtained based on the Ruppeiner's Geometry is taken as the research object. If the curvature scalar is not 0, there exists thermodynamic interaction in the system; if the curvature scalar is diverging, the system has partial phase change. Hence, the phase change structure of thermodynamic system unit can be revealed by the divergence of the curvature scalar of the Ruppeiner's Geometry. Totally different from the Weinhold's Geometry, the Ruppeiner's Geometry was derived from the thermo dynamic fluctuation theory, of which the physical picture is clearer than that of the Weinhold's Geometry, and greatly promoted its application.

Based on the observed results of phase structure of 4D RN-AdS black hole of the predecessors, this paper mainly analyzes the thermodynamic geometrical properties of 4D RN black hole. Some scientists applied the Ruppeiner's geometry in BTZ black hole research, and found that the thermodynamic curvature diverges in extreme case of black hole, having revealed the phase change of BTZ black hole with 0 dimension; besides, Aman et al. applied it in a series of black holes; some ones also applied it in multi-dimensional black hole, and found the Kerr black hole (6D), and revealed that the Ruppeiner curvature diverges in case of unstable black hole, while the Ruppeiner's geometry of RN black hole of any dimension is even. Chamblin et al. had made great contributions, having drawn a conclusion on phase structure of RN-AdS black hole similar to that on Van der WaalsMaxwell liquid-gas system. First, the $Q-\phi$ diagram of RN-AdS black hole thermodynamics is highly similar to the P-V diagram of Van der Waals - Maxwell liquid-gas system. Second, selecting different system parameters from the two phases of RN-AdS black hole to describe TV is just like selecting different sequential parameters to describe the energy density of the two phases of Van der Waals-Maxwell liquid-gas system. If the Ruppeiner metric is taken as a function of entropy, mass and electric charge, RN black hole metric is straight, and the curvature scalar is 0 ; if the Ruppeiner metric is taken as a function of entropy, angular momentum and mass, the metric of Kerr black hole is always curved, and the curvature scalar diverges. This reveals that the Ruppeiner metrics of these two kinds of black hole are different.

Besides common black hole researches as mentioned above, the Ruppeiner's Geometry also can be applied in more complicated researches, say, black hole phase transition in the string theory or the modified gravity theory, such as equilibrium space of electrified topology Gauss-Bonnet black hole in AdS space-time and phase structure geometry. It is found that the positions of thermal runaway in the cases that the curvature scalar of the Ruppeiner's geometry diverges and that the electric charge is constant are same.

\section{Thermodynamic Research on Properties of RN Black Hole}

RN black hole refers to static electrified black hole, of which the 4D metric is:

$$
d s^{2}=-f(r) d t^{2}+f(r)^{-1} d r^{2}+r^{2} d \Omega^{2}
$$

Wherein, $d \Omega^{2}$ refers to unit 2D spherical line element; $f(r)$ should meet the condition of $1-\frac{\mu}{r}+\frac{q^{2}}{r^{2}}$, and $\mu / 2=\mathrm{M}$; q refers to the quantity of electric charge of black hole, and M to the ADM mass of black hole. Since RN black hole has two different horizons, namely internal horizon and external horizon, of which the radiuses are $\mathrm{r}-$ and $\mathrm{r}^{+}$respectively, it can be concluded that by expanding the electric charge and the mass:

$$
\mu=r_{-}+r_{+}, q^{2}=r r_{+}
$$

Wherein, $q^{2} \leq \mu / 4$ should be met. Except for the case of $r=0$, if $(r-)=\left(r^{+}\right)$, it is an extreme black hole. According to the area theorem, the entropy is calculated as below:

$$
S=\frac{A}{4}=\pi r_{+}^{2},
$$


Then, according to the law of conservation of energy of black hole, the potential and temperature of black hole horizons can be obtained, namely:

$$
\begin{array}{r}
\phi=\left(\frac{\partial M}{\partial q}\right)_{s}=\frac{\sqrt{r_{+} r}}{r_{+}}=\frac{q}{r_{+}} ; \\
T=\left(\frac{\partial M}{\partial S}\right)_{q}=\frac{r_{+}-r_{-}}{4 \pi r_{+}^{2}} .
\end{array}
$$

From the equations set forth herein, further information of RN black hole state can be learned about, and the state equation is:

$$
\text { (EOS) } \phi=\phi(T, q) \text {; }
$$

As mentioned above, RN-AdS black hole is highly similar to Van der Waals-Maxwell liquid-gas system. To analyze the state parameters of $\phi$ and q of RN-AdS black hole is equivalent to analyzing the state parameters of Van der Waals-Maxwell liquid-gas system, for which further discussions are needed. According to the first law of thermodynamics:

$$
d u=T d s-P d V ;
$$

Wherein, u refers to internal energy of system, $\mathrm{V}$ to volume, and $\mathrm{P}$ to pressure. Through analysis of phase diagram of RN-AdS black hole, it is found that $\phi$ is equivalent to $\mathrm{V}$ in Van der WaalsMaxwell liquid-gas system, and q to $\mathrm{P}$ in the system. On this basis, the phase structure of RN-AdS black hole can be figured out.

For purpose of this paper, the enthalpy $\mathrm{H}$ of $\mathrm{RN}$ black hole is defined as below:

$$
H=M-\phi q
$$

by differentiating, then:

$$
d H-T d S-q d \phi
$$

In this way, the correspondence between RN-AdS black hole and Van der Waals-Maxwell liquid-gas system can be clearly worked out. The enthalpy of RN black hole is defined here, which will be subject to research as thermodynamic potential in the process of discussing properties of black hole.

\section{Probing into Properties of RN Black Hole from A Thermodynamic Geometrical Angle}

For purpose of research, Ruppeiner metric is introduced first. Defining metric is performed to obtain the Ruppeiner metric of RN black hole, namely:

$$
\boldsymbol{g}_{a b}^{R}=\frac{\partial^{2}}{\partial X^{a} \partial X^{b}} S(H, \phi) \text {, wherein, }(\mathrm{a}, \mathrm{b}=1,2)
$$

Enthalpy $\mathrm{H}$ is taken as thermodynamic potential for the research. The parameter $\phi$ is not adopted as a generally defined extensive quantity, but as a new quantity defined based on Ruppeiner metric, which applies to the geometrical metric of Ewinhold as below. Based on the preceding part of this paper, a new metric will be obtained by merging equations, of which the curvature scalar is:

$$
\widehat{R}=g_{a b} R^{a b}=-\frac{r_{+}-r_{-}}{\pi r_{+}\left(3 r_{-}-r_{+}\right)^{2}}
$$

It is found that if RN black hole becomes an extreme black hole, namely if ( $\mathrm{r}+$ ) equals to ( $\mathrm{r}-)$, the curvature scalar vanishes to 0 . Yet, in most cases curvature scalar is not equal to 0 . If $r+$ equals to 3 $(\mathrm{r}-)$, the curvature scalar is minus infinity. According to the theory of Ruppeiner metric and the phase change of system, the point of divergence of curvature scalar is Davis phase transformation point. The correctness can be further justified by calculating thermal capacity of electric charge: 


$$
C_{q}=T\left(\frac{\partial S}{\partial T}\right)_{q}=\frac{2 \pi r_{+}\left(r_{+}-r_{-}\right)}{3 r_{-}-r_{+}} ;
$$

If $\mathrm{r}+$ equals to 3 (r-), RN black hole is at the stage of second-order phase transition. Thus, it is concluded that Ruppeiner's geometry is an ideal choice for describing the critical behaviors of RN black hole thermodynamic system.

Similarly, according to the definition of Weinhold's geometry, the curvature scalar of RN black hole can be calculated. Though the point of divergence of curvature scalar obtained via Weinhold's geometry is consistent with Davis phase transformation point, it is unable to describe an extreme black hole with this method. Furthermore, the comparison between Ruppeiner's geometry and Weinhold's geometry shows that there exists no apparent proportional relation between components of metrics of the two methods. In other words, Ruppeiner's geometry and Weinhold's geometry are not conformal.

\section{Conclusion}

To sum up, Weinhold's geometry, which lacks physical interpretation, is seldom adopted in researches on black hole properties, especially on black hole phase change. Weinhold's geometry, however, is related to Ruppeiner's geometry to a certain extent in respect of metric. Many scientists adopt Weinhold metric to calculate Ruppeiner metric, and calculate curvature scalar based thereon. Research properties of different black holes from a thermodynamic geometrical angle, and useful results will be obtained. In actual work, combining thermodynamic geometrical method with traditional thermodynamic geometrical method is feasible. For example, Banerjee et al. adopted thermodynamic geometrical method, in combination with Ehrenfest equation, to research the process of phase change of RN-AdS black hole, to analyze first phase change or second phase change, etc. Thereafter, more and more similar practices occurred, by which it was found that these two methods were not mutually exclusive but compatible. In this paper, useful results are obtained by applying Ruppeiner's geometry. It is to be noted that though Ruppeiner's geometry is relatively superior, it also has certain defects, such as high dependence on thermodynamic potential selection. Besides, how a proper metric is selected is specially stated in Ruppeiner's geometry. Studies show that it is unable to make legendre transformation remain unchanged with either Ruppeiner's geometry or Weinhold's geometry, and even conflicting results are obtained some times. Therefore, this problem should be noted in thermodynamic geometrical research on properties of black hole.

\section{Acknowledgement}

A project of scientific research initial foundation of China West Normal University (08B057).

\section{References}

[1] Yuan Fangfang. Holographic Description of Black Hole Thermodynamics and Gravitation Theory, Beijing University of Technology, 2013.

[2] Wang Bin. Current Situation and Future of Research on Extreme Black Hole Thermodynamics, Journal of Shanghai Normal University (Natural Science Edition), 2000, 29(4): 35-40.

[3] Mo Jiexiong. State Equation of (2+1)D AdS Black Hole in Expansion Phase Space, Journal of Zhanjiang Normal University, 2013, 34(6): 34-39.

[4] Zhang Xin. Horava-Lifshitz Thermodynamic Behavior of Gravitation Theory and Thermodynamic Description of Holographic Dark Energy, Liaoning Normal University, 2013.

[5] Zhang Lichun, Han Fulong, Zhao Ren et al. Statistic Entropy and Nernst Theorem of Dirac Field in Reissner-Nordstrom Black Hole Geometry, Acta Mathematical Scientia, 2003, 23(1): 77-83. 